Que peut-on s'acheter avec l'argent gagné au jeu? Les réponses du Joueur de Dostoïevski, des Voyageurs de l'impériale d'Aragon et de Jours de chance de Philippe Adam

\title{
Natalia Leclerc
}

\section{OpenEdition}

\section{Journals}

Édition électronique

URL : http://journals.openedition.org/sdj/425

DOI : $10.4000 /$ sdj. 425

ISSN : 2269-2657

Éditeur

Laboratoire EXPERICE - Centre de Recherche Interuniversitaire Expérience Ressources Culturelles Education

\section{Référence électronique}

Natalia Leclerc, «Que peut-on s'acheter avec l'argent gagné au jeu ? Les réponses du Joueur de Dostoïevski, des Voyageurs de l'impériale d'Aragon et de Jours de chance de Philippe Adam », Sciences du jeu [En ligne], 3 | 2015, mis en ligne le 22 juillet 2015, consulté le 01 mai 2019. URL : http:// journals.openedition.org/sdj/425; DOI : 10.4000/sdj.425

Ce document a été généré automatiquement le 1 mai 2019.

Tous droits réservés 


\title{
Que peut-on s'acheter avec l'argent gagné au jeu? Les réponses du Joueur de Dostoïevski, des Voyageurs de l'impériale d'Aragon et de Jours de chance de Philippe Adam
}

\author{
Natalia Leclerc
}

1 Si, au-delà du plaisir procuré par l'expérience de la lecture, on envisage la littérature comme une représentation du monde, comme une mimèsis au sens où Paul Ricœur a pu la définir dans Temps et récit, on peut l'aborder comme une manière de modéliser le monde et de le donner à comprendre. Si l'on n'envisage plus les lectures formalistes et structurales comme entrées principales dans les textes, mais qu'on accorde à ceux-ci notre crédit, on peut les lire non pas comme des documents, des témoignages, ou des collections de matériau empirique, mais comme une mise en forme du réel, comme la production d'une réalité fictionnelle qui a quelque chose à nous dire sur le réel dans lequel nous évoluons.

2 C'est à la réalité du jeu que présente la littérature qu'il s'agit ici de s'intéresser. Les romans qui traitent du jeu ou qui mettent en scène des personnages de joueurs engendrent chez le lecteur une réflexion sur le statut de l'argent dans le jeu, sur la particularité de ses fonctions, distinctes de celles que l'argent exerce dans l'échange commercial usuel, celui où la transaction s'effectue par l'intermédiaire d'une monnaie. Trois romans proposent une vision de la passion du jeu telle qu'il est intéressant de les étudier dans une approche comparatiste : Le Joueur, que Dostoïevski rédigea en 1866, Les Voyageurs de l'impériale, qui connut une histoire éditoriale complexe, entre une parution expurgée - à l'insu d'Aragon - en 1942 et une seconde version réécrite en 1965, et Jours de chance, de Philippe Adam, paru en 2011. L'hétérogénéité de ce corpus est frappante : les époques, les langues, les contextes (et les intrigues) sont différents. 
3 Dostoïevski transpose dans son personnage, Alexis Ivanovitch, son expérience personnelle de la dépendance à la roulette. Il avait d'abord projeté de faire de son joueur un personnage de poète fasciné par le risque, mais la menace de la prison pour dettes et les exigences de son éditeur ${ }^{1}$, contraignent Dostoïevski à rédiger Le Joueur dans la précipitation. Outre sa dépendance personnelle au jeu, le roman de Dostoïevski se place dans la filiation d'un ensemble d'œuvres russes traitant du jeu: La Dame de pique, de Pouchkine (1834), plusieurs textes de Lermontov (Bal masqué, Chtoss, La Femme du trésorier de Tambov, Un Fataliste), la pièce de Gogol Les Joueurs (1842). Il semble que l'on puisse admettre l'existence dans la littérature russe d'une histoire de la présence du thème du jeu, et certaines de ses significations ont pu être analysées par Iouri Lotman (2012). Les personnages proviennent d'horizons sociaux contrastés: Alexis Ivanovitch est le précepteur d'une famille noble mais désargentée. La vieille grand-mère représente la noblesse terrienne qui n'a pas encore pris le virage du capitalisme. Mais l'on rencontre également une aventurière, Mlle Blanche, et un aventurier - Des Grieux - dont le patronyme est directement issu du roman de l'abbé Prévôt, Manon Lescaut.

4 Le roman d'Aragon a lui aussi un ancrage autobiographique, à plusieurs titres, et notamment pour la présence du thème du jeu de hasard. Pierre Mercadier est la reconstruction littéraire d'une personne réelle, celle du grand-père maternel d'Aragon, Ferdinand Toucas, qui, après avoir été ruiné au jeu, abandonna sa femme et ses quatre enfants pour ouvrir une maison de jeux à Istanbul. Les catégories sociales sont diverses dans ce roman également, mais varient essentiellement de la petite à la grande bourgeoisie. Ainsi le rapport à l'argent diffère également selon le détenteur et son statut. L'époque à laquelle le roman fut composé et publié tient un rôle important pour la compréhension de ses significations. Les Voyageurs de l'impériale est, entre autres, le portrait de la génération qui a conduit l'Europe à la première guerre mondiale, qui en est responsable, en raison de son individualisme. Rédigé juste avant la seconde guerre mondiale, il a une valeur analogique.

Enfin Jours de chance se distingue, par son écriture et sa composition, des deux précédents. Loin d'être structuré autour de l'intrigue suivie d'un personnage principal, il est composé d'un ensemble de fragments relatant le destin de joueurs heureux. Ces joueurs reparaissent régulièrement et leur histoire se tisse au fil de ces surgissements. Ces bribes d'action se déroulent à une époque contemporaine de la nôtre, dans un univers désenchanté.

6 Pourquoi ces trois romans? Le Joueur est ici considéré comme un pivot dans l'histoire littéraire du jeu de hasard, et pour le corpus envisagé, comme le premier jalon d'un parcours : si le jeu intervenait essentiellement en contexte fantastique dans la première moitié du XIX ${ }^{\mathrm{e}}$ siècle, il s'inscrit à partir de Dostoïevski dans un contexte réaliste, mais en transposant dans celui-ci la notion d'ouverture infinie des possibles qui lui était liée dans la littérature fantastique. De nombreux romans sur le joueur prennent le roman de Dostoïevski comme référence. C'est le cas des Voyageurs de l'impériale, qui tisse un important réseau intertextuel avec Le Joueur, comme Grenouillet (2001) l'a mis en évidence. Les trois textes ont été choisis parce que les joueurs trouvent - ou croient trouver - dans le jeu un sens à leur existence, qui n'en a plus. Ainsi, si leur motivation apparente est l'enrichissement, leur quête est en réalité existentielle et métaphysique. Les personnages sont marqués par la solitude ou l'isolement, et pratiquer le jeu d'argent apparaît comme une solution - qui se révèle illusoire - pour entrer dans une communauté de joueurs, mais aussi pour s'inscrire, par le biais de l'argent, dans un flux d'échange. 
7 Le roman de Dostoïevski Le Joueur présente surtout deux personnages au destin inverse : la grand-mère est prise d'une subite folie pour le jeu, mais l'argent qu'elle flambe constitue en fait une libération; le narrateur, Alexis Ivanovitch, lui, plonge dans ce que nous appellerions aujourd'hui une addiction, reflet de la «passion pathologique » que l'auteur lui-même subit et dont Freud présenta une interprétation psychanalytique ${ }^{2}$ dans son article « Dostoïevski et le parricide » (Freud, 1928). Dans la dernière partie du roman, l'argent n'est plus qu'un prétexte, l'objet qui lui permet toujours de rejouer. Il n'entre presque dans aucun circuit d'échange, maintenant le joueur dans sa solitude. Dans Les Voyageurs de l'impériale, Aragon retrace la vie d'un personnage médiocre et individualiste, Pierre Mercadier, fasciné par l'argent et en particulier par le papier-monnaie tel qu'il fut pensé par Law (et que l'on peut concevoir comme une métaphore de la question de la réalité et de la fiction qui obséda Aragon durant toute son enfance et son existence), qui finit par abandonner sa famille et acheva de dilapider sa fortune, pensant trouver une saveur à son existence qui n'en a jamais eu. Jours de chance de Philippe Adam a la particularité d'être un des rares romans qui s'intéresse à des grands gagnants au jeu. Toutefois, cette série de micro-récits répète le scénario de l'échec de ces gagnants au plan humain, et met en scène l'absurdité de chacune de ces existences.

8 À la lecture de ces romans, on peut donc se demander ce que cherchent les joueurs d'argent tels que les romans de Dostoïevski, Aragon et Adam les élaborent, en confrontant cette quête à ce qu'ils trouvent en réalité, pour enfin replacer cette dynamique dans le cadre d'une réflexion sur la valeur sociale de l'argent du jeu, telle qu'elle est notamment présentée par Georg Simmel.

\section{Ce que cherchent les joueurs d'argent}

\section{Le bien-être}

9 Face à ce corpus, on peut se demander si l'argent gagné au jeu est un cas à part dans l'analyse que Simmel fait de ce concept dans Philosophie de l'argent, ou si au contraire, il en est l'aboutissement extrême. Simmel montre en effet que, de moyen placé dans une série téléologique, l'argent se change en valeur absolue, par sa convertibilité en toute marchandise. Pour lui, posséder de l'argent, c'est posséder un pouvoir pur accompagné d'un sentiment psychologique de puissance, mais également d'une véritable liberté de choix. Cette déification de l'argent, devenu valeur suprême, ouvre d'ailleurs la voie à plusieurs pathologies, comme la cupidité, l'avarice ou le cynisme, que le philosophe décrit.

10 L'argent du jeu, au même titre que l'argent gagné au travail, pourrait être le moyen d'acquérir des biens et des services. Simple médium, l'argent, quelle que soit sa provenance, est l'outil que l'homme a à sa disposition pour se procurer ce qui lui semble nécessaire - que cette nécessité soit réelle ou fantasmée. Simmel présente le caractère instrumental de l'argent au début du troisième chapitre de sa Philosophie de l'argent: «L'argent est l'outil le plus pur [...]: une institution dans laquelle l'individu verse son action ou son avoir, pour atteindre par ce point de passage des objectifs qui resteraient inaccessibles à un effort directement dirigé vers eux. » (Simmel, 1987, p. 243) Ce moyen est aussi pour le sociologue ce qui fait l'humanité de l'homme, qui se distingue à la fois de l'animal, rivé aux besoins immédiats et aux instincts, et de la divinité omnipotente. 
11 Avant de sombrer dans la pratique compulsive du jeu de hasard, le narrateur du Joueur de Dostoïevski se rend d'abord au casino dans l'espoir d'y gagner de l'argent pour Pauline. La jeune femme pourrait avoir une situation si elle épousait le Français Des Grieux, qui lui a demandé sa main, mais qui attend, pour confirmer son offre, que l'oncle de Pauline, le général, lui rembourse ses dettes. Le jeu apparaît comme le seul recours pour Pauline et pour le narrateur, qui ne disposent pas de moyen autre de se procurer de l'argent - et certainement pas les émoluments du narrateur, qui travaille comme précepteur dans la famille du général, mais gagne un salaire trop modeste. Gagner de l'argent au jeu s'inscrit donc dans un plan plus global, doté d'une finalité et même d'une stratégie.

Mais même dans cette situation, où l'argent semble être un simple moyen, et le jeu, la seule solution envisageable pour en acquérir, les distorsions apparaissent. Des Grieux porte significativement le même patronyme que le héros du roman Manon Lescaut de l'abbé Prévost. Indirectement, Pauline est assimilée à Manon, une courtisane que l'on achète. De plus, les motivations du narrateur pour lui fournir cet argent ne sont pas limpides : au début du roman, il se dit lui-même amoureux, et même victime d'aliénation amoureuse à l'égard de Pauline. L'argent du jeu pourrait servir à s'offrir des personnes, à les considérer elles-mêmes comme des moyens plutôt que comme des fins. Enfin, autre zone trouble de cette transaction : le grand départ du narrateur au casino au chapitre XV n'est pas la première mention de cette possibilité d'aider Pauline. Au début du roman, Pauline elle-même profite de son aliénation affective pour demander à Alexis Ivanovitch de jouer pour elle :

Elle m'avait même prévenu que je devrais jouer à sa place, parce qu'il était indécent qu'elle joue elle-même. Au ton de sa voix, j'avais compris qu'il y avait là un souci grave et pas, tout simplement, le désir de gagner de l'argent. L'argent en tant que tel, qu'en avait-elle à faire ? ! [...] Il va de soi que l'humiliation et l'esclavage dans lesquels elle me tient pouvaient me donner (peuvent donner souvent) la possibilité de poser des questions d'une manière grossière, directe. (Dostoïevski, 1991, p. 27)

L'argent comme simple instrument neutre devient rapidement le lieu d'enjeux de pouvoir, et ce qu'il permet de se procurer ne se limite pas à des objets ou des services.

Les personnages de Philippe Adam, dans Jours de chance, sont d'emblée placés dans des situations plus nettement distordues. L'argent gagné au Loto est souvent dépensé, réinjecté dans un circuit de dépenses, mais celles qui sont mises en valeur dans le récit sont d'une nature ciblée: si l'on s'appuie sur la pyramide de Maslow, cet argent satisfait, outre les besoins physiologiques et les besoin de sécurité, qui ne sont pas objet de narration, les besoins situés en haut de la pyramide (appartenance et amour, estime, accomplissement de soi - ce dernier étant à interroger). En effet, le narrateur raconte surtout que cet argent permet de s'acheter des voitures coûteuses, des vacances de luxe, et la plupart des anecdotes soulignent la rupture entre les besoins réels et ce que l'argent gagné permet d'acquérir. Du reste, l'argent est moins orienté sur les objets ou services visés pour soi ou pour autrui que sur les personnes à qui le gagnant fait un cadeau, comme pour se racheter de la faute que constituerait son gain - à moins qu'il ne s'agisse plus nettement d'acheter une personne en tant que telle. Ainsi, à celui qui a été abandonné par sa compagne, son ami Hervé rétorque qu'il n'aura « qu'à la racheter à son nouveau mec. » (Adam, 2011, p. 29) Dans une optique légèrement différente, le narrateur énumère les cadeaux d'anniversaire toujours plus fastueux que cette mère fait à sa fille (Adam, 2011, p. 102). Mais loin de parvenir à acheter son affection, la mère ne récolte que son mépris, jusqu'à ce que la fille devenue jeune fille en vienne aux mains avec elle. L'auteur montre ainsi que l'argent gagné au jeu pervertit les relations humaines, 
notamment en tant qu'il génère l'envie des autres ou une forme de culpabilité chez le gagnant. Il ne parvient donc pas à rester un simple moyen, puisque derrière toute dépense, une fin autre qu'utilitaire se profile et tient à l'humain, aux rapports interpersonnels et aux rapports sociaux. Quant au bien-être matériel, il ne correspond jamais à un besoin essentiel et devient de manière presque systématique une fin en soi. Posséder de l'argent ou des objets de luxe revient au même, les objets étant la simple concrétisation de l'argent qui, au XXI ${ }^{\mathrm{e}}$ siècle, s'est dématérialisé. Ces achats appartiennent à la catégorie des dépenses ostentatoires analysées par Norbert Elias (2008) dans La Société de cour et ont pour seule fonction l'affichage de son appartenance à une forme d'aristocratie. Le gagnant au jeu ne s'est pas procuré son argent ou ses biens grâce au travail, il prétend à la reconnaissance d'un statut analogue à celui de la noblesse. L'un des intérêts du roman de Philippe Adam est d'apporter un éclairage très différent de celui de la sociologie, et en particulier de l'étude de Michel Pinçon et Monique Pinçon-Charlot (2010), Les Millionnaires de la chance. Les sociologues montrent au contraire que les grands gagnants, majoritairement issus de catégories socio-professionnelles modestes, ont du mal à assumer la quantité d'argent gagnée, notamment vis-à-vis de leur entourage. Ils se trouvent plus à l'aise entre grands gagnants, dans des rencontres organisées par La Française des Jeux, qui leur apprend notamment à gérer leur fortune nouvellement acquise. Le groupe ainsi formé ne ressemble guère à une nouvelle aristocratie, en tout cas en ce qui concerne l'ostentation des richesses, qui tend à être évitée.

\section{L'appartenance à une communauté}

15 L'ostentation se fait par définition en direction d'autrui. À ce titre, l'argent du jeu acquiert à nouveau le statut de moyen. Il réintègre un circuit d'échange, lui qui semblait marqué par une irrépressible gratuité, mais les échanges effectués grâce à lui sont connotés et l'argent ainsi dépensé y reste marqué en tant que provenant du jeu, pour reprendre la terminologie de Viviana Zelizer. Ce circuit n'est pas celui des biens et des services, mais celui des rapports sociaux. Acheter des objets ostentatoires sert à intégrer une communauté, celle des gagnants. Mais cette communauté représente aussi une forme de marginalité.

En amont de la communauté des gagnants, jouer, miser de l'argent, c'est aussi payer pour être admis dans une communauté. Bien qu'individuels, les jeux décrits dans les textes - la roulette, le loto - sont en réalité des jeux moins solitaires que les jeux de stratégie. Pour jouer à la roulette, il faut nécessairement se rendre dans un lieu consacré, le casino, payer un droit d'entrée dans l'espace lui-même, puis payer pour entrer à une table. Dostoïevski fait plusieurs fois allusion à ce phénomène. Dans les premiers chapitres du Joueur, il décrit le fonctionnement du casino, et notamment sa vie intérieure, la société qui le compose, montrant qu'il s'agit d'un microcosme, doté de ses filous et sa police, de sa hiérarchie sociale, la motivation au jeu des gentlemen n'ayant rien à voir avec celle de la plèbe, ses caractéristiques culturelles, puisqu'un Allemand ne joue pas comme un Russe. Dans cet agrégat d'individus qu'est le casino, des liens se tissent de manière originale: le mimétisme agit lorsqu'un joueur adopte la stratégie d'un autre, qu'il copie parce qu'il la croit gagnante. Des systèmes de charité et de supériorité se mettent en place, notamment lorsqu'un joueur, comme la grand-mère, remporte une forte somme et se voit approché par ceux qui viennent quémander auprès de lui. De même, au chapitre xv, le narrateur, heureux au jeu, reçoit plusieurs fois le conseil de quitter le casino avant que la roue ne 
tourne et qu'il ne perde tout. Jeu solitaire, la roulette fait graviter des individus autour d'elle, et les fait entrer en contact par le biais de l'argent qui met de l'huile dans les rouages sociaux et crée des interactions.

17 Pour sa part, le joueur de loto est peut-être seul derrière son écran de télévision, dans l'attente des résultats, mais lorsqu'il mise, son argent entre dans un grand circuit de redistribution, comme le constate un des personnages de Philippe Adam :

Parmi les gens que je croisais, il y en avait forcément qui avaient financé ma cagnotte, des gens qui, sans le savoir, m'avaient donné les vingt ou trente euros qui n'étaient vraiment plus rien du tout pour moi mais qui aujourd'hui leur manquaient peut-être pour régler une facture d'électricité ou fêter dignement l'anniversaire des enfants. (Adam, 2011, p. 86)

Il s'inscrit donc dans une communauté plus large que celle des gagnants. Il appartient à la communauté des joueurs entre lesquels l'argent s'échange en circuit relativement clos. Le jeu produit donc la synthèse d'une communauté dans la marginalité, d'un microcosme dont les rouages sont actionnés par l'argent.

\section{Un sens à l'existence}

19 Le joueur paie pour entrer dans une communauté car la solitude lui apparaît absurde. De manière plus générale, le jeu de hasard et en particulier d'argent a pour fonction d'aider le joueur à trouver un sens à son existence, qui lui en semble dépourvue. Ce pour quoi le joueur paie, c'est donc aussi pour obtenir le verdict de l'oracle, voire le jugement, si le jeu est conçu comme une ordalie - ce que Marc Valleur a montré dans nombre de ses travaux, et notamment dans son article "Les Chemins de l'ordalie» (Valleur, 2009). Le jeu a en effet à voir avec la divination : les objets - cartes, dés - sont les mêmes, et le résultat d'un jeu de hasard est bien, formellement, une sentence.

20 Ce pour quoi le joueur paie, enfin, c'est pour éprouver le sentiment de puissance et de liberté décrits par Georg Simmel :

[...] la nuance spécifique du "pouvoir", en ce qui [...] concerne [l'argent], est poussée

à l'extrême : c'est véritablement un simple possible, au sens d'un à-venir sans lequel

le présent que nous avons en main n'aurait pas de sens; mais c'est aussi un réel

"pouvoir", au sens qu'on est absolument certain de la réalisation de cet avenir.

(Simmel, 1987, p. 290)

21 Notons toutefois la situation spécifique du joueur qui peut (a la possibilité et le pouvoir de) jouer de l'argent, c'est-à-dire en gagner, mais aussi en perdre.

22 Ainsi, dans Le Joueur, le personnage de la grand-mère peut au premier abord sembler pathétique : elle flambe toute sa fortune et n'a plus qu'à mourir - ce qui, étant donné son grand âge, était annoncé dès le début du roman. Mais envisagée sous un autre angle, sa crise de passion pour la roulette prend un tour plus nettement existentiel : la grand-mère se sait proche de la mort, et sait que ses héritiers s'en réjouissent et n'attendent que cela pour régler leurs propres dettes. Lorsqu'elle flambe sa fortune, elle leur fait un ultime pied de nez, et se venge de leur rapacité. À défaut de donner un sens à sa vie, parvenue à son terme, elle donne un sens à sa mort, pirouette facétieuse ou règlement de compte envers les vautours intéressés et obséquieux qui planent autour d'elle.

23 Les scènes de jeu, durant lesquelles elle perd rapidement sa grande fortune après avoir bénéficié d'une chance insolente lors de sa première venue au casino, semblent donc constituer le verdict d'un échec, cruellement répété jusqu'à l'extinction complète de sa 
fortune. Mais lues sous l'angle de la vengeance, ces scènes représentent un verdict destiné, en réalité, à ses héritiers, et un triomphe de la puissance de la grand-mère puissance annoncée à son arrivée et apparemment battue en brèche par ses pertes au jeu, mais en fait magnifiée par ces mêmes pertes dans un retournement dialectique, où pouvoir flamber sa fortune est un signe de triomphe.

Mercadier, le personnage principal des Voyageurs de l'impériale, souffre également d'une existence dépourvue de sens - même si le narrateur fait en sorte de ne susciter aucune empathie chez le lecteur pour ce personnage faible et mesquin. Lorsqu'il quitte sa femme, ses enfants et son métier, Mercadier part à Venise puis à Monte Carlo où il rencontre le jeu de hasard, lui qui est déjà adepte du boursicotage. Le jeu de hasard devient alors une indication à suivre pour continuer à vivre une existence qui a depuis longtemps perdu toute saveur. D'ailleurs, il est notable que, dans la première partie du roman, alors qu'il vit encore avec Paulette, il essaie de redonner du piquant à sa vie par le jeu en Bourse. La spéculation lui permet d'éprouver des petits frissons, et surtout de mentir à sa femme sous ses yeux :

Peut-être eût-il fait en d'autres circonstances un joueur de roulette. Mais la vie lui rendait plus facile le plus abstrait, le plus nu de tous les jeux: la spéculation boursière. On passe des ordres. On attend. On suit la cote de la Bourse. [...] Ainsi, le matin, en prenant le petit déjeuner, des beurrées dans le café au lait, avant de partir pour le lycée, Pierre Mercadier lisait son journal sans avoir l'air de rien. Et, en réalité, à ce moment-là, sous le nez de Paulette, il jouait, il jouait effectivement. [...] N'aimant plus Paulette, il la trompait ainsi. (Aragon, 1972, p. 59)

Toutes les formes de jeu envisagées par Mercadier sont des espaces de liberté et de puissance, et jouer avec l'argent du ménage est aussi une manière pour lui d'alléger le joug que lui impose Paulette, sa sottise, avant de s'en libérer complètement.

Toutefois, mise à part la grand-mère, qui gagne une belle mort, les autres personnages de ce corpus ont un destin peu enviable. La distance est grande entre ce qu'ils cherchaient dans le jeu d'argent, et ce qu'ils y ont trouvé.

\section{Ce que les joueurs d'argent trouvent dans le jeu}

\section{Une aliénation}

La fiction s'empare de la passion du jeu en insistant sur sa dimension pathologique sans en atténuer la gravité. La littérature scientifique, et notamment le DSM-IV (1994), met en avant les repères que les «joueurs pathologiques " y trouvent, car à sa manière, la compulsion au jeu donne une charpente à leur vie : Christian Bucher et Valleur ont par exemple souligné l'assurance que ces joueurs compulsifs trouvent dans le scénario de la perte, plus sécurisant que l'aléa de l'existence (Bucher et Valleur, 1997). Elle impose à l'existence du sujet une régularité, qui est celle de la perte, un déroulement sans surprise. En apparence, le joueur est mu par l'espoir de gagner, mais en réalité, il chercherait l'assurance, la réassurance de la perte. Tout se passe comme si la dépendance portait moins sur le jeu lui-même que sur la certitude inlassablement répétée de cette ruine.

Le rituel prend donc le dessus sur le résultat du jeu en lui-même, et l'argent apparaît comme le moyen de continuer à réaliser ce rituel, qui a à voir avec la sacralité. Les instruments des jeux de hasard sont les mêmes que ceux de la divination, et le jeu peut être interprété comme une tentative d'accéder à la connaissance de son destin, dans la 
mesure où le hasard est une autre désignation du destin ou de la Providence. Le traitement de la temporalité met cette ritualité en évidence. Le temps du jeu d'argent est un temps circonscrit, de même que l'espace ludique est un espace spécifique. Ils ne se confondent ni avec la temporalité et la spatialité de la vie quotidienne, ni celles du travail, et on pourrait même s'interroger sur leurs rapports avec le loisir. Dostoïevski et Aragon montrent que la société contient les passions pathologiques des joueurs, en leur imposant des horaires. L'argent a beau être liquide, échapper entre les doigts des joueurs, il se heurte à la porte du casino tant que l'heure de jouer n'a pas sonné :

Il faut dire que chaque jour l'attente de l'heure à laquelle on pouvait jouer avait très rapidement pris pour Pierre Mercadier le caractère d'une souffrance. Au vrai, toute habitude chez lui avait ce caractère, et il lui était arrivé d'attendre le facteur avec une inquiétude presque intolérable, à la campagne, à Sainteville par exemple, alors qu'il savait qu'il n'y aurait pas de lettre pour lui dans le courrier, uniquement à cause de la répétition quotidienne du phénomène facteur. (Aragon, 1972, p. 416)

Le temps ne s'achète pas, ou du moins, Mercadier ne peut s'offrir d'accélérer le temps, et le rapport de puissance est inversé : avoir de l'argent, vouloir le mettre en jeu crée un sentiment douloureux d'impuissance lorsque satisfaire ce besoin est impossible. Mais la souffrance ainsi aiguisée peut avoir comme résultat pour le sujet de décupler la rage de jouer son argent lorsqu'il parvient enfin à intégrer le cadre ludique.

Mercadier est, on l'a vu, un personnage qui considère que le monde et les relations humaines ne sont fondés que sur l'argent. Pourtant, il ne va pas jouer pour s'enrichir et son rapport au jeu évoque les ressorts psychologiques que l'on attribue aujourd'hui au stéréotype du joueur pathologique. Alors que le lecteur a déjà un aperçu convaincant de sa mesquinerie, le narrateur le montre se complaisant à se faire passer pour un écrivain, lui qui a rédigé un texte sur John Law, l'inventeur du papier-monnaie, ou à accepter d'être considéré comme un original par l'Anglais Hugh Walter Travelyan. Le narrateur joue de son ambiguïté, et, par le jeu des points de vue, brouille les pistes, ce qui rend difficile la tâche de se prononcer sur le véritable sens de sa pratique du jeu, si toutefois il en est une. Suite à la scène à Venise, où il a compris que « la loi morale du monde, c'est le jeu » (Aragon, 1972, p. 410), et où le lecteur a l'impression qu'il attribue à cette pratique un sens métaphysique - qui donnerait au personnage une grandeur tragique dont il était jusque-là dépourvu - il reparaît dans toute sa médiocrité à Monte Carlo, médiocrité qui va jusqu'à mystifier Travelyan, peut-être pour se débarrasser de ce gêneur et préserver sa solitude. Pour Travelyan, « dans le jeu, il y a le jeu et il y a l'argent... Il y a ceux qui jouent pour l'argent. L'argent brouille tout, absolument tout... L'argent est une chose bestiale. Je peux bien dire, moi... pour qui la question ne se pose pas... ( (Aragon, 1972, p. 419) Malgré l'arrogance de ce riche jeune homme gâté, qui n'a rien à craindre, son analyse lie intimement le jeu à la prise de risque, dont l'argent misé n'est que la matérialisation. Considérer que le gain d'argent est une finalité vient entacher, pour Travelyan, la pureté du jeu de hasard : « Même quand on ne joue pas pour l'argent, on joue à cause de l'argent. N'est-ce pas, c'est une chose si incompréhensible, si effrayante, si puissante... » (Aragon, 1972, p. 420) Mais manifestement, Mercadier ne veut pas avoir affaire à lui, et évacue la discussion en se disant proche des petites gens, de ceux donc, qui jouent pour l'argent. Il nie qu'il pratique ou s'intéresse au jeu pour des raisons existentielles, et fait croire à ce fantasque Anglais qu'il appartient à la catégorie des joueurs intéressés. Peut-être est-ce vrai, ou peut-être est-ce une manière pour lui d'écarter Travelyan. Face à cette alternative, l'attraction dont Mercadier est victime change sensiblement de signification, 
puisque dans le premier cas, il confirme sa bassesse, tandis que dans le second, le personnage prend une épaisseur tragique.

31 Le personnage de joueur de Dostoïevski n'a pas cette ambiguïté : à la fin du roman, il est devenu le joueur « compulsif » qui joue pour jouer, et de manière significative, c'est déjà dans un dialogue avec un Anglais, Mr. Astley, que le narrateur le met en évidence. Notons que les personnages du roman de Dostoïevski réapparaissent dans de nombreux textes postérieurs dédiés au jeu de hasard (ainsi, le général russe intervient dans Vingt-quatre heures de la vie d'une femme, de Zweig) et que l'Anglais d'Aragon pourrait être une réminiscence de celui de Dostoïevski :

Vous vous êtes encroûté, remarqua-t-il, non seulement vous avez renoncé à la vie, à vos intérêts personnels et publics, à votre devoir d'homme et de citoyen, à vos amis (et vous en avez eu, jadis), non seulement vous avez renoncé à tout autre but que celui de gagner, vous avez même renoncé à vos souvenirs ; [...] vos rêves, vos rêves d'aujourd'hui, vos désirs les plus profonds ne vont pas plus loin que pair et impair, rouge, noir, les douze du milieu, etc., etc., j'en suis persuadé! (Dostoïevski, 1991, p. 203)

Et en effet, la pièce qu'il trouve au fond de sa poche devrait lui permettre de s'offrir un dîner, mais il préfère retourner à la roulette. Pour ce personnage, le jeu d'argent est une passion qui s'alimente elle-même, puisque les gains au jeu ne servent à aucune finalité extérieure au jeu et retournent là d'où ils viennent. La présentation du jeu comme un geste pleinement autotélique, marqué par une fermeture qui n'est pas sans rapport, on l'a $\mathrm{vu}$, avec la dimension rituelle, sinon sacrée de l'univers et de la pratique ludiques, est récurrente dans l'histoire littéraire du jeu. Elle fait écho aux travaux des anthropologues et des historiens qui, comme Huizinga, dans Homo ludens, associent la structure hermétique du jeu à une structure sacrée, séparée de l'univers de la vie quotidienne. Cette dimension sacrée est en outre soutenue par la ritualité du geste ludique, qui vient sinon confirmer du moins alimenter la perception du jeu comme activité liée au sacré.

\section{La solitude}

Cette fermeture va de pair avec la solitude à laquelle le joueur d'argent se confronte inévitablement, lui qui cherchait la communauté de ses pairs. Bien que Mr Astley lui apprenne que Pauline est effectivement amoureuse de lui, Alexis Ivanovitch ne revient pas à l'aliénation amoureuse qui le liait à elle au début du roman. Il est désormais solitaire, coupé des hommes par sa propre volonté, et à la limite de l'enfermement psychologique - ce que ses séjours réguliers en prison pour dettes matérialisent. Si, à la fin du roman, il affiche l'intention de « renaître à nouveau, ressusciter. [...] redevenir un homme » (Dostoïevski, 1991, p. 209), les dernières lignes contredisent cet élan vers la libération.

La solitude de Mercadier a une résonance plus politique au sens large. À travers ce personnage, Aragon dénonce, dans ce roman publié pour la première fois intégralement en 1948, mais écrit et partiellement publié avant la deuxième guerre mondiale, l'« individualisme forcené » (Aragon, 1972, p. 744) en tant que responsable de la première guerre mondiale. C'est au nom de cet individualisme que Mercadier abandonne sa famille, son métier, qu'il part à Venise et Monte Carlo. Ce personnage est un cynique sans envergure :

Les subtilités ne font que déguiser la nature profonde, la nature commune de ces liens, de ces chaînes. 
L'argent... Il n'y a pas de sentiments, il n'y a que l'argent.

Ce qui unit Pierre à Paulette c'est l'argent. (Aragon, 1972, p. 306) minable, il ne peut rien faire d'autre que fréquenter la maison de passe Les Hirondelles, et y devenir l'objet des rêveries romantiques de sa tenancière Dora avant de finir sa vie piteusement. Ses derniers jours constituent le summum de l'avilissement de cet homme qui a voulu la puissance, la liberté à tout prix : victime d'une crise d'apoplexie, il devient aphasique, totalement dépendant de Dora qui le tyrannise avec son amour, et ne parvient plus qu'à articuler un mot difficilement audible, " po-li-tique ». Cet homme qui a méprisé la politique, agacé ses interlocuteurs à force d'ignorance des grands événements mondiaux du début du $\mathrm{xx}^{\mathrm{e}}$ siècle, meurt au moment où il se rend finalement compte de la responsabilité de chacun dans la marche de la société, et où il a décidé de renouer avec sa famille. Le jeu d'argent fait partie des facteurs qui l'ont coupé du monde de manière irrémédiable. Cet argent n'appartient à aucun circuit d'échange et condamne qui le manipule à ne plus pouvoir réintégrer la société.

En outre, cet argent n'est pas échangeable contre tout, il n'a pas la valeur absolue que Simmel lui attribue. La solitude des gagnants dans le roman de Philippe Adam en rend le titre ironique : Jours de chance. La grande fête organisée pour le nouvel an est désertée par tous les invités et Jean-Baptiste exprime tout haut ce que le lecteur a compris depuis plusieurs pages : « les gens n'aiment pas les riches, les riches n'ont pas d'amis.» (Adam, 2011, p. 33) Ce constat peut aussi virer à la paranoïa, ou à une forme de lucidité si l'on admet que les gagnants au loto gagnent une clairvoyance dont les autres humains sont dépourvus : "J'ai commencé à me méfier de tout le monde. Aujourd'hui je sais que les gens ne sont pas bons les uns pour les autres. Une fois cette évidence admise, on comprend qu'avant on s'énervait pour rien.» (Adam, 2011, p. 87) À moins, au contraire, que l'argent, comme l'amour, ne rende aveugle :

Mes possessions, mes dépossessions n'ont aucune importance, ce qui compte, c'est autre chose, et quand les gens viennent à moi, c'est sans doute plutôt pour ma façon d'être, ma façon de parler et de réfléchir, ou peut-être aussi pour ma façon de m'habiller, je ne sais pas. (Adam, 2011, p. 117)

Cette solitude est liée à un élément structurel du jeu d'argent : si - au loto du moins l'argent qui circule est bien celui des autres joueurs, si, quand l'un gagne, cela signifie bien que l'autre perd, il faut avoir à l'esprit le fait que cet argent ne transite pas directement d'un joueur à un autre, mais passe par la «banque ", et que c'est elle qui ensuite redistribue les richesses. Peut-être faut-il voir dans cette instance tierce au loto le maillon surnuméraire dans la chaîne des échanges, qui empêche la relation directe entre les joueurs, et qui les enferme dans leur solitude.

Le joueur en vient à ne plus avoir d'échanges avec autrui qui ne soient essentiellement monétaires. Les romans étudiés montrent la manière dont les autres échanges humains se restreignent peu à peu. Et réciproquement, Simmel pour sa part montre que les relations monétarisées sont fondamentalement impersonnelles, et que si l'invention de l'argent a augmenté la liberté individuelle, elle a aussi transformé les relations communautaires en relations sociétaires - ce qui éclaire d'un jour nouveau les relations entre individus dans les casinos notamment, qui ne constituent plus des micro-communautés, mais des microsociétés. 


\section{La vanité}

39 Le jeu d'argent ne frappe pas seulement du sceau de la vanité les relations humaines: c'est toute l'existence qui semble menacée. Les biens que les gagnants au loto peuvent s'acheter apparaissent comme des cache-misère destinés à masquer la vacuité de leur existence. Ne plus avoir besoin de travailler pour vivre prive l'individu du sentiment d'utilité sociale :

J'avais du temps libre. Pour certains, c'est une chance et même le but d'une vie. Moi, je n'ai pas su quoi en faire, j'étais un retraité de trente ans, je traînais sans avoir vraiment envie d'aller d'un côté plutôt que de l'autre, et à présent je suis devenu tout ce que je n'aimais pas, je joue aux mots fléchés. (Adam, 2011, p. 89)

La recherche des expériences connotant le luxe, autre usage de cette richesse mal acquise, est tout aussi désespérante :

Il faut être resté plusieurs semaines en peignoir, regardant la télévision à longueur de journée sans quitter le lit ni les salons trop chauffés de son hôtel particulier sous prétexte que Venise est humide, il faut avoir trempé ses lèvres dans un verre de château-léoville-poyferré 1855 et l'avoir trouvé dégueulasse [...] pour comprendre que tout le monde ne court pas dans la même catégorie et qu'il ne suffit certainement pas d'être riche pour que s'ouvrent devant soi les grands bras du grand monde. (Adam, 2011, p. 119-120)

41 Quel est le véritable gain que remporte alors le joueur? Si l'on s'appuie sur les deux personnages du corpus qui meurent à la fin de leur roman, la grand-mère et Mercadier, on s'aperçoit que les relie le sentiment d'avoir vécu. La grand-mère meurt satisfaite d'avoir accompli quelque chose de grand: priver des êtres sans morale d'un héritage indu. Quant à Mercadier, s'il a une fois éprouvé des sensations fortes qui se détachent sur le fond de sa vie sans relief, c'est surtout à Venise - contrairement au personnage précédemment cité de Philippe Adam - qu'il éprouve un sentiment existentiel, lors de la partie de cartes qui l'oppose à Angelo, le prétendu frère de Francesca, la jeune fille qui fait semblant d'être séduite par lui pour l'attirer dans un guet-apens. Angelo le défie donc au jeu, mais à la fin de l'épisode, on ne saura pas exactement quelles étaient les intentions du jeune homme : s'il s'agissait de détrousser Mercadier, il a échoué. Le premier pari, peut-être destiné à l'impressionner, portait sur la tête de Mercadier, qui l'a gardée. Progressivement, les deux joueurs se mettent à parier de l'argent, et c'est Mercadier qui détrousse le garçon. Lorsque celui-ci propose de parier deux diamants qu'il a volés, la prise de conscience de Mercadier est subite :

Est-ce qu'il n'avait pas volé, lui aussi ? Paulette et les gosses? Tout son argent était une rapine. L'argent d'ailleurs est toujours de la rapine. Ici, enfin, les mensonges s'évanouissent dans la loi du jeu, qui transforme tout, comme le feu qui rend pur... (Aragon, 1972, p. 403)

Pour le personnage, le monde, la société sont frappés d'immoralité, et seul le jeu, censé être gratuit, permet à Mercadier d'atteindre, sinon la moralité, du moins l'amoralité. Mais cette catharsis qui lui est apportée par le jeu ne peut être totale tant que l'argent est en jeu - elle ne pourrait éventuellement l'être, et encore, que si le jeu était parfaitement gratuit - et si le jeu semble ouvrir la voie à une société utopique, cette utopie - comme toutes les utopies du reste - se révèle bientôt angoissante : « Les cartes s'abattent et les dés roulent, la propriété s'évanouit, la malédiction du travail est mise en déroute, l'argent naît du hasard ou s'y absorbe : rien n'est plus stable, monde fuyant... Ah, le jeu, toutes les morales 
battues par le jeu!» (Aragon, 1972, p. 403) C'est alors que Mercadier entrevoit la voie de la libération, voie qu'il ne suit finalement pas :

[...] il ne s'agissait plus pour Pierre Mercadier que de la grande justification de soimême... Que de se prouver que l'argent qui avait dominé sa vie était moins fort que lui-même, qu'il pouvait bafouer l'argent, bafouer tout au monde, jeter sur la table sa vie comme les hommes de jadis dans la bataille, miser sur une carte à la fois la catastrophe et sa rédemption, la preuve de sa liberté... Trembler pour l'argent et le nier, se sentir à la fois plus haut que la perte et prêt à s'y engloutir. " (Aragon, 1972, p. 403-404)

Nier la valeur de l'argent aurait permis de donner un sens élevé à son existence, et c'est ce que Mercadier essaie de faire à Monte Carlo, mais il n'a pas le courage de se jeter à corps perdu dans la bataille et n'accomplit que médiocrement, sur le mode mineur, cet ambitieux programme.

Dans Le Joueur, on trouve un épisode analogue: suite à son gain prodigieux au casino, initialement destiné à Pauline - qui le refuse, le narrateur est happé par la demimondaine Blanche, qui l'entraîne à Paris, où elle se charge de dilapider sa fortune en dépenses somptueuses. Le lecteur a d'abord l'impression que le narrateur se fait flouer, jusqu'à ce qu'il comprenne en réalité que les dépenses de Blanche permettent à Alexis Ivanovitch de se délester plus rapidement de l'argent qu'il a gagné et qui l'empêche moralement de retourner au jeu. Lorsque ses gains se sont évanouis, il retrouve une légitimité pour retourner jouer. Le narrateur aurait été un joueur tragique s'il avait réussi à se détacher complètement de l'argent, mais le rapport ambigu qu'il entretient avec lui entache la grandeur du personnage, dont la vie n'apparaît ni pleine, ni complètement vide, mais simplement médiocre - humaine somme toute.

\section{Quelle est la signification sociale de l'argent du jeu?}

Considérer le joueur sous l'angle de son humanité plutôt que sous celui de sa pathologie permet de déplacer le regard sur son comportement, sur son rapport à l'argent. Sur le terrain littéraire, il s'agit en l'occurrence de considérer un personnage comme la projection d'une figure humaine plus que comme un type, ou un stéréotype - forme de configuration littéraire à laquelle pourrait tendre le joueur au titre de sa maladie ou de son rapport à l'argent justement, comme l'avare en est un. Mais si l'on n'envisage plus le joueur comme un malade existentiel, et qu'on le considère en tant qu'un humain comme les autres, comment définir son rapport à l'argent? Viviana Zelizer dénonce la "puissante idéologie de notre temps» selon laquelle «l'argent serait un instrument unique, interchangeable et absolument impersonnel - [...] l'essence même de la rationalisation inhérente à notre civilisation contemporaine. » (Zelizer, 2005 [1994], p. 27) L'argent du jeu n'est pas plus neutre que n'importe quel autre argent, et on peut se demander, à la manière de Zelizer, quelle est la nature de son marquage.

\section{II n'est ni un simple moyen ni une simple fin}

Si l'argent du jeu était un simple moyen, il serait caractérisé par sa neutralité, ce qu'il n'est pas. Il n'est pas dépensé comme on dépense l'argent de la vie quotidienne, ni même l'argent du loisir. La grand-mère se paie une mort digne. Alexis Ivanovitch se paie son rituel compulsif. Pierre Mercadier se paie son éphémère mais réel sentiment de liberté. 
Les gagnants de Jours de chance s'offrent des objets de luxe dont ils n'ont que faire. Ce qu'ils acquièrent est donc soit impalpable, dans les trois premiers cas, soit palpable mais absurdement évanescent au sens où cela ne correspond à aucune utilité réelle, dans le cas des personnages de Philippe Adam. Paradoxalement, la détermination symbolique de l'argent du jeu pourrait être la gratuité. Ainsi, le bénéfice tiré de ces transactions ne s'évalue pas en termes d'avoir. D'ailleurs, on l'a vu, l'argent n'est pas non plus une fin en soi dans le jeu, puisqu'il n'y a pas à proprement parler d'objectif d'enrichissement, ou si désir d'enrichissement il y a, il se révèle être une façade, ou un objectif qui s'anéantit de lui-même lorsqu'il se réalise. Quel est donc cet objet qui n'est ni fin ni moyen, dont l'acquisition ne perdure pas, et qui disparaît et reparaît de manière presque spectrale?

Si le statut ontologique de l'argent du jeu pose problème, c'est précisément parce que le jeu lui-même a à voir avec l'être. Le fait de jouer donne aux personnages le sentiment d'avoir vécu, et le flux d'argent apparaît comme le flux d'une énergie vitale, ou plutôt survitale si l'on peut tenter un néologisme. Les joueurs ne sont en effet pas des vivants, mais des survivants, et ce qu'ils achètent avec l'argent du jeu, c'est une apparence de vie. La thématique du joueur situé à la limite entre la vie et la mort est intervenue à plusieurs reprises dans la littérature du XIX ${ }^{e}$ siècle, qui a vu le développement de la figure du joueur existentiel. Dans La Dame de pique, nouvelle rédigée par Pouchkine en 1834, et qui est une référence dans la littérature sur le jeu de hasard, en particulier pour Dostoïevski, le personnage central de la vieille comtesse est comparé à un automate, et le motif de la mécanique est exploité dans la signification donnée au jeu du pharaon. Le jeu d'argent est un automatisme qui s'enraye et entraîne le personnage principal, Hermann, dans la folie, lui qui croyait pouvoir se rendre maître de ces rouages. Le jeune ingénieur allemand, qui pensait dominer le hasard et donc faire fortune, perd beaucoup plus que ce qu'il possédait au départ.

Le personnage emblématique de la vieille femme reparaît dans Le Joueur, avec la grandmère, mais aussi dans Les Voyageurs de l'impériale, avec la vieille lady Hutchinson. Elle a ceci d'emblématique qu'elle représente le joueur idéal, sur lequel on peut faire des calculs statistiques, car elle est dotée d'une fortune illimitée et peut donc jouer un nombre illimité de coups. Ironiquement, et de manière tout à fait explicite, Aragon attribue sa fortune à la vente d'armes : l'appétence humaine pour la guerre étant sans fin, la vente de mitraillettes rapporte à lady Hutchinson une fortune sans cesse renouvelée et jamais mise en péril, et la conduit donc toujours à gagner, " au bout du compte, au bout de la nuit. » (Aragon, 1972, p. 413) Pour Mercadier, ce « monstre » est aux confins de l'humanité :

Il éprouve en elle un achèvement extraordinaire de la destinée humaine. Il voit en elle l'image dernière de ce que l'argent fait d'un être de chair et de sang. [...] Est-ce qu'elle aime vraiment le jeu, elle qui s'y rend chaque soir comme à un travail, avec son cœur malade, ses jambes gonflées, ses yeux voilés, ses reins rompus? Elle l'aime et elle ne l'aime pas : elle n'a pas le choix de jouer ou de ne pas jouer, c'est tout ce qu'elle peut faire. Physiquement, moralement aussi sans doute. C'est tout ce qui disqualifie encore le temps pour elle. C'est tout ce qui le peuple, et c'est tout ce qui le tue. (Aragon, 1972, p. 414)

Jouer n'est pas un luxe, ni un caprice, ni une dépense insensée, c'est l'unique possibilité qui s'offre au joueur, pour qui l'argent intervient alors comme un principe vital. Il n'est ni une fin ni un moyen mais une nécessité.

50 C'est aussi pour cela que lorsque le joueur joue, il n'emporte pas une catégorie spécifique d'argent, celle qu'il aurait mise de côté pour le jeu, mais il mise tout ce qu'il a, et la grandmère du Joueur, qui n'était pas venue à Roulettenbourg dans l'intention de jouer et qui 
n'avait jamais franchi le pas d'un casino auparavant, en vient à changer tous ses avoirs, à mettre en jeu tous ses titres de propriété, à se dépouiller intégralement, puisque l'argent destiné au jeu transcende toutes les catégories et tous les marquages.

\section{L'argent du jeu et l'individu}

51 L'argent du jeu est donc intrinsèquement lié à l'individu. Ce n'est pas un attribut, une possession, mais une part du sujet. Comme la peau de chagrin dans le roman éponyme de Balzac, peau que le héros Raphaël de Valentin acquiert d'ailleurs en sortant d'une salle de jeux, cet argent est le miroir de l'homme, le reflet de son existence. Il figure la vie que le joueur dépense, tout en espérant la gagner, vie qui rétrécit inéluctablement et que le joueur essaie de regonfler en l'alimentant d'argent.

52 Dans le jeu de hasard et d'argent, le joueur est face à lui-même, car l'autre que constitue la banque n'est pas un véritable partenaire ni adversaire de jeu. Et dans ce face-à-face avec lui-même, le sujet ne se nourrit que de ces flux d'argent, flux dont la signification n'est pas intrinsèquement monétaire, liée à une valeur, mais fonctionnent comme des flux d'énergie vitale. Mercadier, à Monte Carlo, comprend cette situation singulière du joueur :

Il sent ce qui est le lien de ces êtres sans lien, de ces gens qui ne se parlent pas, qui se voient à peine, et que l'heure ramène chaque soir à la même fièvre, à la même amertume, à la même passion. Il sait que c'est la solitude. Il sait enfin qu'il a pénétré dans le monde étrange de la vraie solitude, et qu'il n'en sortira plus. (Aragon, 1972, p. 415)

Le joueur est dans une situation paradoxale, celle qui consiste à appartenir à une communauté de solitaires. Dans cette structure qui pourrait se nier elle-même, l'argent s'est substitué à ce qu'il attend de ses congénères. Sa fonction sociale est ici de se substituer à la société.

Le joueur est devenu autarcique, autonome, autotélique - comme le jeu lui-même. Ce circuit fermé de l'homme à lui-même risque à tout moment d'imploser et c'est pourquoi le joueur cherche tout de même une issue pour que l'énergie qui l'agite ne le détruise pas. Cette énergie, matérialisée par l'argent, ne doit pas non plus être évacuée de manière incontrôlée et le constat de ce gagnant de Jours de chance est aussi désabusé que gros de menaces: «Dans les premiers temps, on ne sait pas quoi faire, on a des rêves et des ambitions d'avant, on a d'anciens désirs qui ne collent plus au pouvoir tout neuf qu'on nous a mis dans les bras.» (Adam, 2011, p. 15) Les menaces semblent se concrétiser quelques pages plus loin - sans que l'on sache si c'est le même joueur qui parle : "On pense longtemps à ce qu'on a gagné. Après on ne pense pas, on a juste la fièvre, l'envie de mordre un cheval ou d'aller dans l'arène s'affronter aux cornes d'un taureau. » (Adam, 2011, p. 18) Ce joueur, ce gagnant semble tellement débordé par l'argent de son gain, qui représente pour lui un déluge d'énergie vitale, que le remettre en jeu apparaît comme la meilleure solution, une solution qui permet de prendre un risque, d'éprouver une intense sensation de vie, d'affronter le taureau.

\section{Conclusion}

Les œuvres littéraires étudiées ici mettent en évidence le gouffre qui sépare ce que les joueurs cherchent dans le jeu et la réalité de ce qu'ils y découvrent. Elles ont été choisies 
car elles constituent des exemples représentatifs de ce mécanisme qui fonde l'écriture des œuvres traitant du jeu de hasard : le jeu est à même de nourrir une dynamique narrative, car il se développe lui-même comme une narration. Et les retournements de situation et épisodes déceptifs qu'il occasionne se prêtent efficacement au récit.

Dans ce gouffre entre l'attente et la réalité se joue également la redéfinition de la signification de l'argent. Pour ces joueurs, l'argent n'est pas un moyen, du moins pas dans un circuit d'échange traditionnel : il n'est pas montré prioritairement comme moyen de se procurer des biens ou des services, et ceux que le joueur achète tout de même répondent à d'autres besoins que les besoins primaires. Mais il n'est pas non plus une finalité, puisque cet argent semble brûler les doigts des joueurs. À moins qu'il ne faille dire qu'il est sa propre fin, puisque l'argent du jeu est immédiatement réinvesti dans le jeu. Pourtant, l'argent permet bien aux joueurs, d'obtenir quelque chose : le sentiment, parfois fugitif, d'avoir vécu. À une existence terne, ces joueurs opposent l'existence justifiée par le jeu.

57 Ainsi, l'argent du jeu ne serait pas échangeable contre tout, comme le montre Simmel, mais uniquement contre certains biens et services qui se rapportent à ses usages, comme le montre Zelizer. Il permettrait de s'offrir ce qui a le plus de prix pour l'homme, à savoir le sentiment d'être vivant - même s'il doit pour cela passer par la perte de soi et même la mort.

En revanche, ce sentiment - qui est peut-être une des formes du bonheur - ne règle pas le problème de la solitude fondamentale du joueur, thème que les trois œuvres étudiées exploitent pour sa portée psychologique ou métaphysique dans le cas de Dostoïevski, politique dans le cas d'Aragon qui dénonce l'individualisme, ou sociale dans le cas de Philippe Adam qui s'intéresse aux configurations formées par les grands gagnants et leur entourage. La circulation de l'argent dans le cadre du jeu n'instaure alors aucune communauté, aucune société - tout au plus quelques associations fugaces. Si l'essence de l'argent tient à la cohésion du groupe, l'argent du jeu, lui, n'a à voir qu'avec l'individu, qui trouve le moyen dans le jeu de ne pas se laisser étouffer par son argent.

\section{BIBLIOGRAPHIE}

ADAM P. (2011), Jours de chance, Paris, Gallimard, « Verticales ».

ARAGON L. (1972), Les Voyageurs de l'impériale [1940/1942], Paris, Gallimard, « Folio ».

BALZAC H. (1974), La Peau de chagrin [1830], Paris, Gallimard, « Folio classique ».

BUCHER C. et VALLEUR M. (1997), Le Jeu pathologique, Paris, PUF, « Que sais-je ? ».

DOSTOÏEVSKI F. M. (1991), Le Joueur [1866], Arles, Actes Sud, « Babel », traduction française d'A. Markowicz.

ELIAS N. (2008), La Société de cour [1969], Paris, Flammarion, « Champs essais ».

FREUD S. (1994), « Dostoïevski et le parricide » [1928], préface aux Frères Karamazov, Gallimard, Folio. 
GOGOL N. (1998), Les Joueurs [1842], trad. A. Markowicz, Paris, Actes Sud.

GRENOUILLET C. (2001), « Le vertige du jeu dans Les Voyageurs de l'impériale », Lectures d'Aragon : Les Voyageurs de l'impériale, sous la direction de Luc Vigier, Presses Universitaires de Rennes, p. $163-176$.

HUIZINGA J. (1988), Homo ludens, essai sur la fonction sociale du jeu [1938], Gallimard, « Tel ».

LERMONTOV M. (1992), Bal Masqué [1835], trad. André Markowicz, Imprimerie Nationale éditions.

LERMONTOV M. (1985), La trésorière de Tambov [1838], dans Euvres poétiques, éd. Efim Etkind, L'Âge d'Homme, « Classiques slaves ».

LERMONTOV M. (2003), Un héros de notre temps (le chapitre Un fataliste) [1841], éd. Déborah LévyBertherat, GF Flammarion.

LERMONTOV M. (2007), Chtoss [1841], dans Lermontov, Odoïevski, Titov, Récits fantastiques russes, trad. Sophie Benech, Corti, « Romantiques ».

LOTMAN Y. (2012), « La Dame de pique et le thème des cartes et du jeu de cartes dans la littérature russe du début du XIX ${ }^{\mathrm{e}}$ siècle » [1975], trad. N. Leclerc, Le Cercle herméneutique, $\mathrm{n}^{\circ} 18-19$, Vrin.

PINÇON M. et PINÇON-CHARLOT M. (2010), Les Millionnaires de la chance. Rêve et réalité, Payot. POUCHKINE A. (1999), La Dame de pique [1834], dans La Dame de pique et Les Récits de feu Ivan Pétrovitch Belkine, Paris, Le Livre de poche, « Classiques de poche », traduction française de D. Seseman.

PRÉVOST A. (1972), Manon Lescaut [1831], Paris, Gallimard, « Folio classique ».

SIMMEL G. (1987), Philosophie de l'argent [1900], Paris, PUF, « Quadrige ».

VALLEUR M. (2009), « Les chemins de l'ordalie », dans Topique, 2009/2, n 107, p. 47-64.

ZELIZER V. (2005), La Signification sociale de l'argent [1994], Paris, Seuil, « Liber ».

\section{NOTES}

1. Stellovski, qui lui donne trois mois pour lui fournir un manuscrit, faute de quoi il aura pendant neuf ans libre jouissance de son œuvre. La même année, Dostoïevski travaille sur Crime et châtiment.

2. Notons toutefois que le sentiment de culpabilité que Freud prétend mettre en évidence n'est pas du tout évident chez le narrateur du Joueur, et invalide soit la lecture psychanalytique, soit la portée autobiographique du roman.

\section{RÉSUMÉS}

Si la motivation apparente des joueurs de hasard est le gain financier, leur motivation réelle est souvent moins intéressée et plus existentielle. La littérature met ce trait en valeur, notamment depuis Dostoïevski, avec Le Joueur, et encore au xxI ${ }^{\mathrm{e}}$ siècle, dans Jours de chance, de Philippe Adam. Entre ces deux romans, Les Voyageurs de l'impériale d'Aragon montre un héros médiocre, 
Mercadier, qui aurait pu trouver un sens à son existence dans le jeu s'il n'était pas détruit par l'individualisme. En effet, dans les textes étudiés, l'argent du jeu n'entre dans aucun circuit d'échange, ne permet aux personnages d'intégrer aucune communauté. Il circule bien sous forme de flux, mais en circuit fermé, n'alimentant que les joueurs et leur passion.

The apparent motivation of gamblers is financial benefit. But their real motivation is often less linked to financial interest than to existential questions. Literature brings this point out, especially since Dostojevski's Gambler, and until now, in the 21st century, in Jours de chance, written by Philippe Adam in 2011. Between these two novels, Aragon's Les Voyageurs de l'impériale shows a mediocre heroe, Mercadier, who could have found a meaning to his life through gambling, if he were not destructed by individualism. Actually, the money of gambling is not included in any trade channel, does not allow to the individual to enter any community. It circulates as a flow, but in a closed channel, and it only sustains the gambler and his passion.

\section{INDEX}

Mots-clés : communauté, individu, moyen, fin, nécessité, quête existentielle, absurdité, jeu compulsif

Keywords : community, individual, means, finality, necessity, existential quest, absurdity, addiction

\section{AUTEUR}

NATALIA LECLERC

UBO / UEB, EA 4249 HCTI 